\title{
Kaon Productions Off Nucleons And The Structure Of Baryon Resonances
}

\author{
Zhenping Li \\ Physics Department, Peking University \\ Beijing, 100871, P.R. China
}

March 11, 2017

\begin{abstract}
The recent investigations in the chiral quark model show that kaon productions of nucleons play an important role in understanding the structure of baryons. The evidences of a third $S_{11}$ resonance in the second resonance region and two narrow states around $2 \mathrm{GeV}$ suggest a set of the molecular type baryons with the hidden strangeness. Confirming these states requires further theoretical and experimental studies of the strangeness production.
\end{abstract}

There have been considerable recent progress in the investigation of baryon resonances in the meson photoproductions. New experimental data [1] for the $\eta$ photoproduction in the threshold region have been published. These data, in particular the data from the Mainz accelerator MAMI, played a very important role in studying the structure of the resonance $S_{11}(1535)$. On the theoretical side, a new approach based on the chiral quark model has been developed[2] for the meson photoproductions. Comparing to other models at the hadronic levels, the quark model approach to the meson photoproductions introduces the quark and gluon degrees of freedom into the reaction mechanism, and it relates the photoproduction data directly to the spin flavor structure of baryon resonances. Here, we would like to highlight some important features that one could learn from the forthcoming kaon production data at TJNAF and the Bonn accelerator ELSA.

An important feature that we have learnt from the $\eta$ photoproduction off nucleons is the enhancement of the resonance $S_{11}(1535)$ and the suppression of the resonance 
$S_{11}(1650)$ in the $\eta N$ channel. In Ref. [3], we showed that this phenomenon can not be explained in the framework of the constituent quark model. The solution of this problem might come from the existence of a third $S_{11}$ resonance in the second resonance region. We indicated that there are considerable circumstantial evidences suggesting the presence of a third $S_{11}$ resonance with mass $1.7 \sim 1.8 \mathrm{GeV}$ and width around 0.2 $\mathrm{GeV}$, which certainly can not be accommodated by the quark model. Moreover, the data from SPHINX collaboration in $p+C$ coherent diffractive productions [4] show two new states,

$$
\begin{array}{r}
X(2000) \rightarrow \Sigma^{0} K^{+}, \quad M_{X(2000)}=1996 \pm 7, \quad \Gamma_{X(2000)}=99 \pm 21 \\
X(2050) \rightarrow \Sigma^{*}(1385) K^{+}, \quad M_{X(2050)}=2052 \pm 6, \quad \Gamma_{X(2050)}=35 \pm 29,
\end{array}
$$

in which the masses and widths are in the unit of $\mathrm{MeV}$. The resonances with such small width at $2 \mathrm{GeV}$ are unlikely to be normal $q q q$ states. Notice that the threshold energies for $K^{*} \Lambda$ and $K^{*} \Sigma$ productions are 2007 and $2084 \mathrm{MeV}$ respectively. A common feature for the $S_{11}$ resonances in the second resonance region and the states $X(2000)$ and $X(2050)$ is that the masses of these states are just below the threshold energies of the kaon and $K^{*}$ productions. This suggests that a set of molecular type baryons with hidden strangeness, $K \Lambda$ or $K \Sigma$ state for the $S_{11}$ resonances in the second resonance region, $K^{*} \Lambda$ for the $X(2000)$ and $K^{*} \Sigma$ for the $X(2050)$, may indeed exists bellow the threshold energies of Kaon and $K^{*}$ productions in addition to the well known $\bar{K} N$ candidate $\Lambda(1405)$. The $K \Lambda$ or $K \Sigma$ quasi bound state was first proposed for the resonance $S_{11}(1535)$ in Ref. [5], while the interpretation of the $K^{*} \Lambda$ and $K^{*} \Sigma$ states for the $X(2000)$ and $X(2050)$ was suggested in Ref. [6]. In Ref. [3] we pointed out that a pure $K \Lambda$ or $K \Sigma$ configuration for the resonance $S_{11}(1535)$ is inconsistent with the data for the electromagnetic form factor of this resonance, and it should be strongly mixed with the normal $q q q S_{11}$ states.

How the $K \Lambda$ or $K \Sigma$ bound state is mixed with normal $q q q S_{11}$ states has not been investigated theoretically. However, the kaon productions would be very important channels to study the structure of these resonances experimentally. Notice that the masses of the $S_{11}$ resonances are sandwiched between the threshold energies of the kaon and the $\eta$ productions, the enhancement of the contributions from the $S_{11}$ resonances are expected in the $\eta$ and kaon productions. This is particularly true for the $\eta$ photoproduction in the threshold region, where the dominance by the contributions from the resonance $S_{11}(1535)$ is well established. A similar behavior should be expected for the kaon photoproduction; our investigations [7] in the $\gamma N \rightarrow K \Sigma$ reactions found that the resonance $S_{11}(1650)$ is indeed enhanced in the threshold region of the reaction $\gamma n \rightarrow K^{-} p$. Thus, the presence of the third $S_{11}$ resonances could be tested in the kaon production experiments. Moreover, as the new states $X(2000)$ and $X(2050)$ are just below the $K^{*} \Lambda$ and $K^{*} \Sigma$ threshold energies, these states are expected to be enhanced 
in the reactions $\gamma N \rightarrow K \pi \Lambda$ and $\gamma N \rightarrow K \pi \Sigma$, as there is no pomeron exchange to contaminate the cross sections. Unfortunately, there are few data available for these reactions to either confirm or refute the presence of these two states. Therefore, the experimental and theoretical studies of the $K \pi$ productions should become one of the top priorities in the strangeness productions. It is very interesting to note that the preliminary data from ELSA[B] indeed have some hints of resonance structures in the 1.7 GeV region of the reaction $\gamma p \rightarrow K^{+} \Lambda$ and around $2.0 \mathrm{GeV}$ region in both reactions $\gamma p \rightarrow K^{+} \Lambda$ and $\gamma p \rightarrow K^{+} \Sigma^{0}$, which might correspond to the third $S_{11}$ resonance and the resonances $X(2000)$ and $X(2050)$. Of course further experiments with better precision, in particular the data for the polarization, are needed. Furthermore, the data in $\pi N \rightarrow K \Lambda$ or $\pi N \rightarrow K^{+} \Sigma$ are also required so that these new states could be fully established.

Thus, understanding the reaction mechanism of kaon productions has become increasingly important. There has been considerable recent progress in understanding the kaon photoproductions in the traditional isobaric models [9] and in the newly developed chiral quark model[2]. An important feature from the quark model approach in the kaon production via $\gamma N \rightarrow K \Sigma$ is that the resonances $F_{37}(1950), F_{35}(1905), P_{33}(1920)$ and $P_{31}(1910)$ belonging to 56 multiplet in the quark model play very important role in the reaction $\gamma N \rightarrow K \Sigma$. Better data in these reactions could provide us important information on the structure of these resonances as well.

In conclusion, the high precision data for kaon photoproductions will provide us very important insights into the structure of baryon resonances that could not be possible from the pion photoproductions, and establish the molecular baryons with the hidden strangeness. It may also help us to resolve the puzzle with the $S_{11}$ resonances in

the second resonance region. Without doubt, the strangeness productions will be an important and exciting field in the near future.

The author acknowledges the collaborations with R. Workman, Wei-Hsing Ma and Zhang Lin on the works presented here. Discussions with B. Saghai and R. Schumacher are gratefully acknowledged. This work was supported in part by Peking University.

\section{References}

[1] S. Dytman et al., Phys. Rev. C51, 2170(1995); J. Price et al., Phys. Rev. C51, R2283(1995); B. Krushe, et al., Phys. Rev. Lett. 74, 3736(1995).

[2] Zhenping Li, Ye Hongxing and Lu Minghui, "An Unified Approach To The Meson Photoproductions Off Nucleons In The Quark Model" To Appear on Phys. Rev. C. (1997). 
[3] Zhenping LI, and R. Workman, Phys. Rev. C53, R549(1996).

[4] SPHINX Collaboration, S.V. Golovkin et al., Z. Phys. C68, 585(1995).

[5] N. Kaiser, P.B. Siegel, and W. Weise, Phys. Lett. B362, 23(1995).

[6] R. Schumacher "Evidence For And Against The States Hidden Strangeness States Near 2 GeV", Submitted to Phys. ReV.

[7] Zhenping Li, Ma Wei-Hsing and Zhang Lin, Phys. Rev. C54, R2171(1996).

[8] D. Menze, in this proceedings.

[9] J. C. David, C. Fayard, G.H. Lamot, and B. Saghai, Phys. Rev. C53, 2613(1996), and references therein. 